\title{
Evolution dans l'exploitation des évacuateurs de crues et rénovations récentes sur les barrages d'Electricité de France
}

\author{
Marc Lefranc \\ Adjoint au chef de la Division Génie Civil \\ du Service de la Production Hydraulique \\ d'ÉLECTRICITÉ DE FRANCE
}

\section{A - GÉNÉRALITÉS}

\section{Rappel de l'approche générale d'E.D.F.}

Le rapport C.I.G.B. sur « La détérioration de barrages et réservoirs" permet une étude statistique assez complète. En résumant, il est fondé de déclarer que les deux causes principales de rupture sont : la submersion et les problèmes liés aux fondations.

La moitié des ruptures par submersion est survenue avant 1930 alors que seul le quart des ouvrages actuels était en service. La grande majorité de ces ruptures correspond à des barrages dont les évacuateurs de crues avaient été sous-dimensionnés, soit par méconnaissance de l'hydrologie, soit par imprudence des projeteurs. Cette cause de rupture a sensiblement diminué depuis 1930, mais il convient de rappeler qu'en matière de dimensionnement des évacuateurs, on peut améliorer la situation des barrages pour tenir compte de la connaissance et des techniques.

Plusieurs facteurs sont à l'origine de la réhabilitation ou du réaménagement des évacuateurs de crue d'un barrage :

- une meilleure connaissance de l'hydrologie et des méthodes de traitement statistique (GRADEX) nous assure une détermination plus précise des valeurs des débits moyens ou instantanés de crues extrêmes;

- une expérience d'exploitation alliée à une compétence plus affinée permet de déceler sur des ouvrages anciens des imprudences de conception ;

- une réglementation administrative de plus en plus vigilante vis-à-vis de la sécurité des biens et des personnes qui impose des seuils de protection et des garanties de fonctionnement quasi-normal dans des circonstances de plus en plus exceptionnelles.

Un seul de ces facteurs suffit à envisager des travaux de mise en conformité des ouvrages d'évacuation des crues, mais bien souvent les circonstances sont telles que plusieurs facteurs concourent à la décision, y compris celui toujours présent de la modernisation des installations et de leur entretien.

Electricité de France est confronté avec ce problème depuis de nombreuses années et la politique suivie dans ce domaine ne souffre aucune équivoque : en matière d'ouvrage d'évacuation des crues, il y a mise en conformité obligatoire afin d'assurer la sécurité. Actuellement, plus d'une douzaine d'ouvrages sont modernisés ou en cours de modernisation.

\section{Différents types d'évacuateurs de crues de barrages}

Deux familles d'évacuateurs de crues se distinguent selon la position de leur entonnement par rapport au niveau normal de la retenue :

- les évacuateurs de surface, les plus répandus,

- les évacuateurs de demi-fond ou de fond qui fonctionnent normalement en charge,

(auxquels il serait bon d'ajouter, pour être exhaustif et pour mémoire, les digues fusibles).

Pour le problème qui nous intéresse, nous considérerons uniquement les évacuateurs de surface. Ils se subdivisent en plusieurs types différents.

- Les seuils déversants sur l'ouvrage ou indépendants des
barrages

Leur fonctionnement hydraulique est régi par l'application des lois de l'écoulement fluide sur les seuils et représente les solutions les plus naturelles, simples et fiables. Elles ne nécessitent aucun appareillage et le couple débit/niveau s'équilibre automatiquement. 


\section{LEFRANC}

Les inconvénients liés à ce choix concernent particulièrement :

- la nécessité d'avoir un niveau amont variable, quelquefois au détriment de la capacité utile,

- l'obligation de disposer topographiquement des lieux nécessaires à l'implantation linéaire d'un tel déversoir et de son écoulement à l'aval. Cette contrainte limite bien souvent les possibilités d'équipement.

- Les ouvrages vannés qui peuvent eux-mêmes être intégrés au barrage ou indépendants, ces derniers réalisés à l'air libre ou en souterrain.

Le critère principal qui différencie leur écoulement est la section (pris au sens large) de contrôle du débit, soit à l'amont (vanne), soit à l'aval (section de mise en charge).

Les avantages ou inconvénients sont a contrario de ceux du type précédent :

- maintien d'un niveau amont constant,

- caractéristiques plus élevées leur permettant une insertion plus facile dans le site,

- nécessité d'un contrôle/commande très sûr, très fiable, très précis, induisant un contrôle et un entretien permanent (une fausse manœuvre peut créer une crue artificielle d'autant plus préjudiciable qu'elle est inattendue).

Ce thème est développé en détail dans l'exposé de M. BISTER.

\section{Causes du réaménagement de ces ouvrages}

Les raisons d'intervention pour la réalisation de travaux sur les évacuateurs de crues peuvent se classer en plusieurs familles :

\subsection{Dégradations par le temps et le fonctionnement}

Nous trouverons, sans ordre de classement :

- désorganisation du béton de structure par l'action chimique des eaux, l'action des rigueurs climatiques (en particulier le gel des bétons),

- l'action de maladies du béton comme les phénomènes de gonflement,

- le mauvais comportement en exploitation par usure (compte tenu de la vitesse et des matériaux de charriage), l'érosion due à la cavitation sur l'ouvrage même ou à l'aval),

- l'obsolescence du matériel d'équipement électromécanique liée parfois à une grande difficulté d'entretien.

\subsection{Fonctionnement défectueux en exploitation}

Dans cette catégorie, nous trouverons principalement :

- Incapacité de certains évacuateurs par leur conception de faire face aux charriages, des bois en particulier. L'accu- mulation de ces matériaux perturbe l'entonnement de l'eau, induit une surélévation du niveau amont et provoque des conditions de fonctionnement en dehors des hypothèses normales de calcul vis-à-vis de la stabilité.

- Incapacité d'évacuer dans des conditions normales les débits passés par les vannes. Cette insuffisance est susceptible d'entraîner des érosions à l'aval des ouvrages conduisant à des instabilités.

- Impossibilité de moderniser par automatisation les dispositifs d'évacuateurs de crues, par suite de la conception générale des installations.

- Risque de blocage par les glaces.

\subsection{Insuffisance des caractéristiques}

C'est principalement dans cette cause que nous trouvons la justification des réhabilitations des évacuateurs de crues. Deux raisons essentielles contribuent à cet état :

\subsubsection{La méconnaissance de la valeur des crues extrêmes lors de l'élaboration du projet}

Beaucoup d'ouvrages sont anciens et ont été construits sur la base des connaissances existant à l'époque et surtout des observations constatées. Le choix du débit de crues peut avoir été fait dans un contexte un peu arbitraire par l'application de règles empiriques. Depuis 1960, deux éléments principaux sont venus améliorer les hypothèses :

- L'existence même de nombreux ouvrages permet de mieux connaitre l'hydrologie et permet l'approche de la connaissance des valeurs extrêmes par les méthodes statiques, à partir d'échantillons de données très fournis.

- La mise au point d'une méthode de réflexion et de calcul (méthode du Gradex) nous procure une approche plus sûre des conditions extrêmes.

\subsubsection{Les modifications successives du seuil de prévention administratif}

Jusqu'en 1970, aucune réglementation propre aux barrages n'existait. Face à une urbanisation grandissante, il y eut une évolution de la notion de risque potentiel lié à la nature même de l'ouvrage et petit à petit naquit une certaine conscience de responsabilité par l'administration. Le contrôle et l'entretien des grands ouvrages fut l'objet, en 1970, d'une réglementation (Circulaire 70/15).

On a ainsi constaté une évolution des critères retenus pour la conception des ouvrages de sécurité et, par conséquence, des contraintes imposées aux maîtres d'ouvrage.

NOTA : Il est exceptionnel qu'une seule des causes soit la seule justification de travaux importants et, au contraire, le maître d'ouvrage constate ou profite de la concomitance de plusieurs causes pour entreprendre des travaux sur les organes de sécurité des barrages que sont les évacuateurs de crues. Il est certain, pour E.D.F., tout au moins, que l'on profitera de la nécessité d'augmenter la capacité des ouvrages d'évacuation pour moderniser ceux-ci, non seulement par le matériel, mais par la conception de leur exploitation, conformément aux règles de contrôle de la sécurité 
de fonctionnement que nous avons érigées en doctrine (Recommandation S.E.B. $\left({ }^{1}\right)$ ).

\section{Processus d'études d'une modification d'ouvrage}

L'opération de réhabilitation ou de réaménagement des ouvrages d'évacuation d'un barrage comporte différentes phases chronologiques indispensables au déroulement logique de l'étude :

\subsection{Etude hydrologique}

La détermination des valeurs extrêmes des débits de crues est un critère fondamental et la précision est une nécessité.

Pour E.D.F., l'étude est conduite selon la « méthode du gradex ", dont le principe est de relier les lois statistiques des débits aux lois statistiques des précipitations. Cette méthode a déjà fait l'objet de nombreuses publications, reprise en particulier dans le Rapport $n^{\circ} 60$ de la Question $63 \mathrm{du} 16^{\mathrm{e}}$ Congrès ICOLD de San Francisco.

L'objectif de cette première phase est de déterminer les valeurs des débits de crues extrêmes en fonction de leur durée de retour.

\subsection{Etude sur modèle réduit hydraulique}

Cette phase a un double but :

- la vérification des caractéristiques maximales des ouvrages en service, après étalonnage du modèle sur les débits de crues fréquents pour lesquels nous disposons de nombreuses informations.

Les phénomènes hydrauliques étant complexes et parfois difficiles à appréhender de façon précise par le calcul (remous, pertes de charge, etc...), nous constatons souvent, pour les ouvrages anciens, un certain décalage entre les débits théoriques calculés et ceux plus proches de la réalité que le modèle nous donne.

Cette phase permet de mettre en évidence l'écart entre les possibilités des ouvrages existants et les caractéristiques minimales imposées par l'administration, et c'est sur cette base qu'est élaboré un avant-projet des nouveaux ouvrages à concevoir ;

- la mise au point d'un avant-projet: le modèle réduit hydraulique permet la vérification et la mise au point des caractéristiques des nouveaux ouvrages, tant sur le plan de l'efficacité hydraulique que sur le plan technique et économique, afin d'assurer leur pérennité. L'étude du dimensionnement et des formes doit être faite dans ce triple contexte.

Pour la réalisation de ces modèles réduits, E.D.F. possède ses propres laboratoires d'hydraulique et fait parfois appel à des organismes privés ou universitaires.

(') S.E.B. : Commission de Sécurité d'Exploitation des Barrages, créée en juillet 1984 à E.D.F., Service de la Production Hydraulique.

\subsection{Etudes détaillées}

L'étude technico-économique sur modèle ayant défini les caractéristiques principales des ouvrages, la mise au point du projet engendre :

- un avant-projet détaillé suivi des études d'exécution,

- une étude d'automatisation des dispositifs, le cas échéant,

- l'élaboration d'une consigne d'exploitation.

Dans sa démarche, en plus de ce qui régit la conception d'un ouvrage neuf, le projeteur doit tenir compte de contraintes liées à l'ouvrage existant, notamment :

\section{- Contrainte de niveau amont}

Cette contrainte a un double aspect :

- Administratif, par le fait que tous les niveaux d'exploitation sont fixés par les cahiers des charges des concessions et toute modification entraînerait une remise en cause des dispositions et une nouvelle enquête, longue et fastidieuse. - Environnement : une modification du niveau des plus hautes eaux peut avoir une incidence sur les propriétés riveraines, ainsi que sur les stabilités géologiques des versants, sans oublier les conséquences d'une influence sur l'hydrogéologie des terrains bordant la retenue.

\section{- Contrainte de niveau aval}

Ceci représente moins une contrainte que des obligations. En effet, l'écoulement du débit extrême de la crue doit s'écouler dans la vallée naturellement, mais nous devons vérifier que cet écoulement n'est pas perturbé par nos ouvrages et, si cela est, prendre des dispositions de protection qui s'imposent.

\section{- Contraintes au niveau de la sécurité}

Pour un évacuateur intégré au barrage, toute modification d'ouvrage peut entraîner un nouvel état d'origine pour le calcul de la sécurité des ouvrages et en particulier vis-à-vis de la stabilité. Il est parfois délicat et difficile d'harmoniser une conception nouvelle d'évacuateurs de crues et un profil existant de barrage sans provoquer des travaux importants d'amélioration de la sécurité de l'ouvrage. Le résultat est souvent l'adoption d'une solution de compromis.

En tout état de cause, il est nécessaire de s'assurer que le comportement de l'ouvrage en exploitation sera conforme aux calculs et les travaux s'accompagneront bien souvent d'une modification ou d'une adaptation par renforcement du dispositif d'auscultation.

\subsection{Réalisation du chantier}

Cette phase classique de construction est l'objet de nombreuses contraintes qui pèsent sur le concepteur de tout projet de réaménagement ou de réhabilitation des évacuateurs de crues d'un ouvrage, surtout lorsqu'il s'agit d'en augmenter les capacités maximales. Elles sont issues de deux faits essentiels : 
- les ouvrages sont en exploitation,

- cette exploitation se fait sous le contrôle de l'organisme de tutelle, avec obligation du respect du cahier des charges particulier à la chute hydraulique et des règles administratives générales applicables à tous ces ouvrages.

Ces sujétions sont l'origine des difficultés rencontrées pour :

- minimiser l'influence des travaux sur l'exploitation de l'ouvrage (arrêt d'exploitation, programme de remplissage de la retenue),

- assurer le passage des crues sans incidence sur la sécurité du barrage et du chantier,
- minimiser les incidences sur la structure du barrage, en particulier prendre les précautions indispensables pour limiter les ébranlements dus aux tirs d'explosifs, lors des terrassements,

- se satisfaire des conditions d'accès, parfois très difficiles, dans une topographie très mouvementée, ne laissant que des possibilités exiguës pour les plates-formes d'installation et de chantier,

- tenir compte, dans la mesure du possible, des incidences sur l'environnement extérieur comme le réseau routier, les utilisations pour les loisirs de la retenue et toutes les contraintes d'environnement.

\section{B - ÉVACUATEURS EN SEUILS DÉVERSANTS INDÉPENDANTS}

\section{Généralités}

Qu'il s'agisse des aménagements de Thuries, Queuilles ou Rochebut, traités en exemples dans cet exposé, le problème rencontré a été le même: sous-dimensionnement des évacuateurs dû à une méconnaissance de l'hydrologie, à la faiblesse des méthodes de calculs de l'époque, à l'absence de modèle hydraulique et à la minimisation des travaux difficiles et des dépenses.

Le résultat conduit à une saturation très rapide du seuil déversant et à un coefficient de débit global (seuil + canal) de l'évacuateur très faible:

$$
\begin{aligned}
& \mathrm{m}=\frac{\mathrm{Q}}{\mathrm{Lh} \sqrt{2 \mathrm{gh}}}=\text { proche de } 0,2, \\
& \mathrm{~L}=\text { Longueur du seuil, } \\
& \mathrm{h}=\text { hauteur de la lame d'eau. }
\end{aligned}
$$

Pour l'amélioration des performances des évacuateurs de Queuilles, Thuries et Rochebut, l'utilisation de modèles hydrauliques a permis de définir en plus des caractéristiques des nouveaux ouvrages, le type d'intervention le plus économique tout en tenant compte des contraintes diverses. Les études et les travaux réalisés nous permettent de tirer les conclusions ou remarques suivantes :

- la longueur des seuils varie généralement peu $(0 \%$ à Thuries, $10 \%$ à Queuilles, $35 \%$ à Rochebut) et les travaux consistent essentiellement à désaturer les canaux d'évacuation ;

- l'augmentation sensible de la section disponible en amont du barrage permet de diminuer les vitesses et zones de turbulences et ainsi obtenir une meilleure mise en vitesse de l'écoulement ;

- le réglage est assuré en jouant sur les conditions aval et l'on minimise les terrassements dans la zone d'appuis du barrage tout en abaissant rapidement la ligne d'eau ;

- une attention particulière est portée aux risques d'instabilité des écoulements et au travail de la cuillère de restitution pour minimiser l'importance et les effets de la fosse d'érosion ;

- il est intéressant de supprimer les revêtements béton du chenal dans toutes les zones de rocher sain, convenablement ancré, lorsque les vitesses ou les irrégularités de l'écoulement ne sont pas excessives; on échappe alors aux problèmes liés au drainage sous ces revêtements dont la pérennité n'est jamais garantie à $100 \%$;

- la réalisation des 3 ouvrages décrits ci-après fait appel à des techniques de minage contrôlées qui permettent d'utiliser des méthodes d'exécution industrielles, et donc de prix raisonnable, tout en conservant un usage partiel de la retenue, fonction des contraintes de niveau des terrassements et du degré de protection souhaité vis-à-vis des aléas hydrologiques.

\section{Barrage de Thuries}

\subsection{Caractéristiques}

Le barrage de Thuries est situé sur le Viaur, affluent de l'Aveyron.

Barrage-poids en béton.

Construction 1917.

Hauteur $31 \mathrm{~m}$ environ.

Longueur $106 \mathrm{~m}$.

Retenue de $6,5 \mathrm{hm}^{3}$.

Hydrologie :

- Bassin versant : $1050 \mathrm{~km}^{2}$.

- DMI centenal : $650 \mathrm{~m}^{3} / \mathrm{s}$.

- DMI millénal : $1000 \mathrm{~m}^{3} / \mathrm{s}$.

— DMI décamillénal : $1300 \mathrm{~m}^{3} / \mathrm{s}$. 


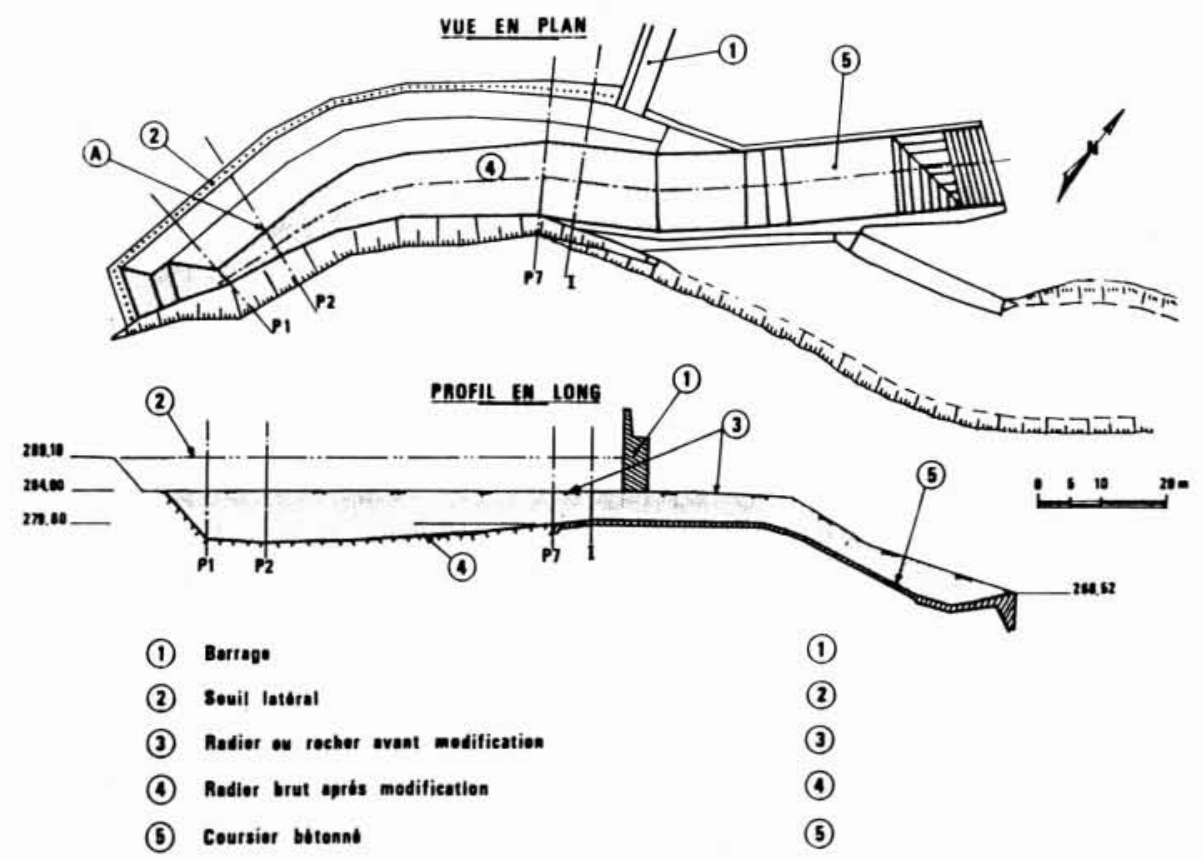

1. Barrage de Thuries. L'évacuateur de crues.

\subsection{Evacuateur de crues initial}

2 seuils libres :

En rive gauche: $\mathrm{L}=90 \mathrm{~m}$ à la sortie 289,70 prolongé par un puits vertical.

$\mathrm{Q}=220 \mathrm{~m}^{3} / \mathrm{s}$ sous PHE mesurées en maquette.

En rive droite $: \mathrm{L}=96 \mathrm{~m}$ à la cote 289,10 prolongé par un canal à écoulement libre.

$\mathrm{Q}=500 \mathrm{~m}^{3} / \mathrm{s}$ sous PHE mesurées sur modèle.

Les essais montrent la saturation de l'évacuateur rive droite par engorgement du canal amont qui noie le seuil, conduisant au débordement du bajoyer aval qui nuit gravement à sa stabilité propre.

Débit évacuable total de $720 \mathrm{~m}^{3} / \mathrm{s}$ à rapprocher du débit nominal instantané de fréquence millénale à $1000 \mathrm{~m}^{3} / \mathrm{s}$.

\subsection{Les options décidées à partir du modèle (fig. 1 et 2)}

En rive gauche: un reprofilage de l'entonnement du puits vertical permet de porter la capacité à $250 \mathrm{~m}^{3}$ sous PHE.

En rive droite:

- Renforcement du seuil amont pour améliorer son coefficient d'écoulement pour limiter les turbulences, et conférer aux filets liquides la forme d'hélices très allongées d'axe parallèle au canal.

- Approfondissement de 4 à $8 \mathrm{~m}$ du canal actuel et élargissement de 0 à $5 \mathrm{~m}$ selon profils pour éloigner dans la courbe, le talus, du seuil déversant.

- Aménagement à l'aval immédiat du barrage d'un coursier bétonné traversant le mur bajoyer R.G., courtcircuitant partiellement la partie aval du canal, reliant directement la partie amont du canal au lit de la rivière.

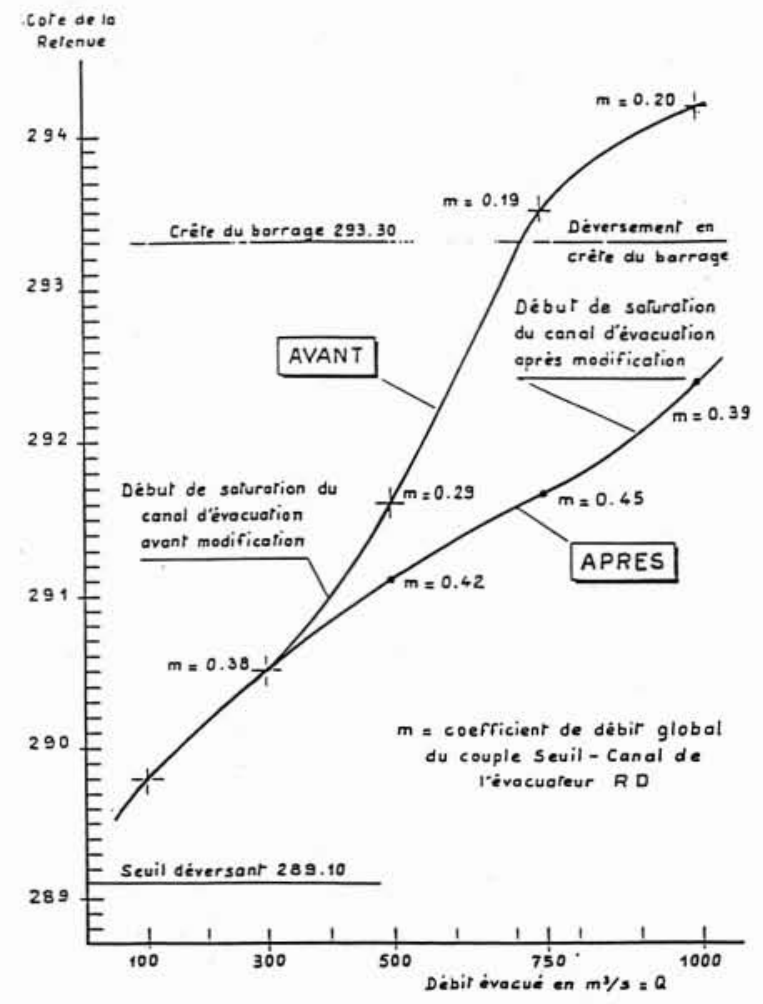

2. Thuries. Courbes $H(Q)$ avant et après modification de l'évacuateur $R_{D}$.

Ces modifications ont porté la capacité de l'évacuateur rive droite à $750 \mathrm{~m}^{3} / \mathrm{s}$ sous PHE.

L'ouvrage est donc mis en état pour évacuer la crue millénale $\left(750 \mathrm{~m}^{3} / \mathrm{s}+250 \mathrm{~m}^{3} / \mathrm{s}\right)$ à l'issue des travaux de 1986. 


\section{Barrage de Queuilles}

\subsection{Caractéristiques}

Le barrage usine de Queuilles est situé sur la Sioule (Affluent de l'Allier), $10 \mathrm{~km}$ à l'aval du barrage des Fades, dans le département du Puy-de-Dôme.

Barrage-poids en béton.

Construction 1905.

Hauteur $32 \mathrm{~m}$ environ.

Longueur $116 \mathrm{~m}$.

Retenue de $6,5 \mathrm{hm}^{3}$.

Hydrologie :

- Bassin versant : $1340 \mathrm{~km}^{2}$.

— DMI millénal : $1300 \mathrm{~m}^{3} / \mathrm{s}$.

\subsection{Evacuateur de crues initial}

En rive gauche: un seuil de $74 \mathrm{~m}$ de longueur, calé à 429,40 NGF déverse dans un canal taillé dans le rocher de la rive, en contournant l'appui rive gauche du barrage. Il se prolonge par un coursier à forte pente et partiellement revêtu de béton.

En rive droite : un seuil de $60 \mathrm{~m}$ de longueur, calé également à 429,40 NGF totalement noyé lors des déversements importants par saturation de son canal.

La partition des débits, mesurés sur modèle réduit, entre les deux déversoirs pour la cote des Plus Hautes Eaux est la suivante :

- débit maximal estimé en rive gauche :

$470 \mathrm{~m}^{3} / \mathrm{s}$

— débit maximal estimé en rive droite :

Total

$\frac{270 \mathrm{~m}^{3} / \mathrm{s}}{740 \mathrm{~m}^{3} / \mathrm{s}}$

Pour mettre cet ouvrage en conformité avec les conclusions d'études et d'observations hydrologiques il a été décidé de porter à $1300 \mathrm{~m}^{3} / \mathrm{s}$ le débit maximal évacuable, correspondant à la crue millénale et à la capacité maximale d'évacuation des vannes de crues du barrage des FADES situé à l'amont.

\subsection{Les options décidées à partir du modèle (fig. 3)}

En rive droite : une limitation à $10 \mathrm{~m}$ de la longueur du seuil existant (limite de saturation du canal), par remblaiement de la partie amont s'appuyant sur un mur de soutènement transversal dans le coursier.

En rive gauche: construction d'un nouveau seuil de $130 \mathrm{~m}$ de longueur au droit de l'ancien ouvrage, élargissement côté falaise et surcreusement du chenal et du coursier aval.

L'ouvrage est donc mis en état pour évacuer la crue millénale $1300 \mathrm{~m}^{3} / \mathrm{s}$ à l'issue des travaux de 1987 .

\section{Barrage de Rochebut}

\subsection{Caractéristiques}

Le barrage de Rochebut (usine de Teillet Argenty) est situé sur le Cher à $15 \mathrm{~km}$ environ à l'amont de Montluçon.

Barrage-poids en béton.

Construction 1909.

Hauteur $48 \mathrm{~m}$ environ.

Longueur $98 \mathrm{~m}$.

Retenue de $20 \mathrm{hm}^{3}$ environ.

Hydrologie :

- Bassin versant : $1600 \mathrm{~km}^{2}$.

- DMI millénial : $1430 \mathrm{~m}^{3} / \mathrm{s}$.

— DMI décamillénal : $2000 \mathrm{~m}^{3} / \mathrm{s}$.

\subsection{Evacuateur de crues initial}

Selon les schémas du début du siècle, courants pour ce type d'ouvrage, le barrage était muni en rive gauche d'un évacuateur de crues constitué d'un seuil latéral et d'un chenal d'évacuation restituant les débits dans une fosse de réception. La forme du seuil retourné dans sa partie amont dans un thalweg.

\subsection{Les études et les options prises (fig. 4 et 5)}

Dès 1940, des correspondances mentionnaient un sousdimensionnement de l'évacuateur de crues.

Entre 1960 et 1962 des calculs du chenal mettaient en évidence une saturation de l'évacuateur à $1000-1100 \mathrm{~m}^{3} / \mathrm{s}$ pour des PHE à 302. Or le service hydrologique E.D.F. de l'époque annonçait un débit maximal de $1400 \mathrm{~m}^{3} / \mathrm{s}$ pour la crue millénale.

Plusieurs ouvrages furent étudiés :

- 1965 : ouvrage additionnel en tulipe.

- Jusqu'en 1969, de nombreuses variantes : (abaissement de la RN, agrandissement de l'ouvrage, ouvrage muni de clapet et enfin 4 siphons de $5 \mathrm{~m} \times 2,5 \mathrm{~m}$ ) ont été comparées. (La plus séduisante sur le plan du coût, les siphons, posait des problèmes de réalisation et d'irrégularité de débit à l'amorçage.)

En 1986-1987, de nouvelles études sur modèle avaient été entreprises sur de nouvelles bases.

Contrairement à ce qui avait été envisagé lors des premières études, l'état du seuil déversant nécessitait sa reconstruction complète.

Une solution de seuil droit s'avéra satisfaisante sur le modèle mais prohibitive dans sa partie centrale. Finalement une forme en baïonnette fut retenue (voir schéma en annexe).

Le nouvel ouvrage d'une capacité de $1430 \mathrm{~m}^{3} / \mathrm{s}$ a été terminé fin 1988. 


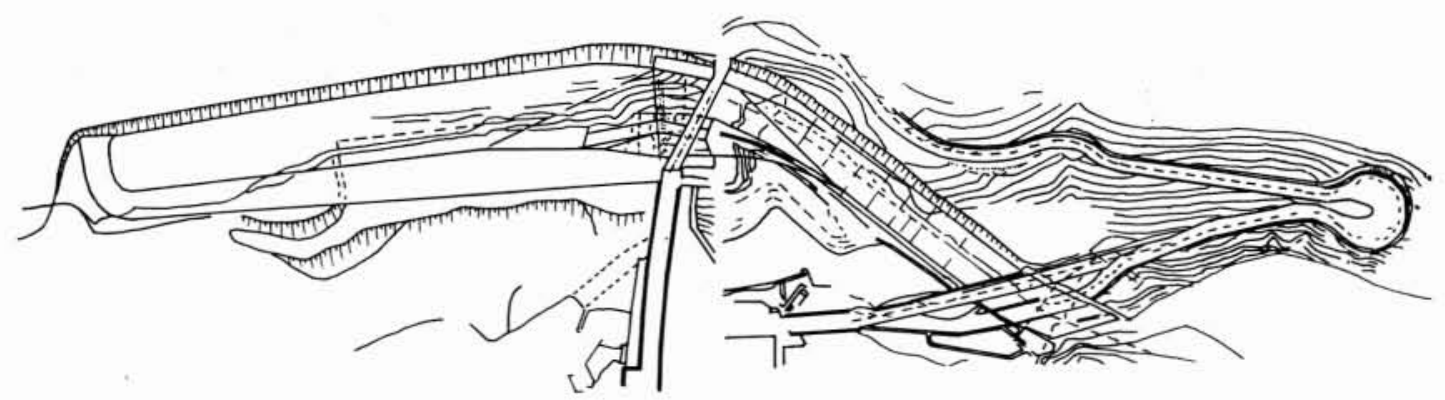

3. Evacuateur du barrage de Queuilles.

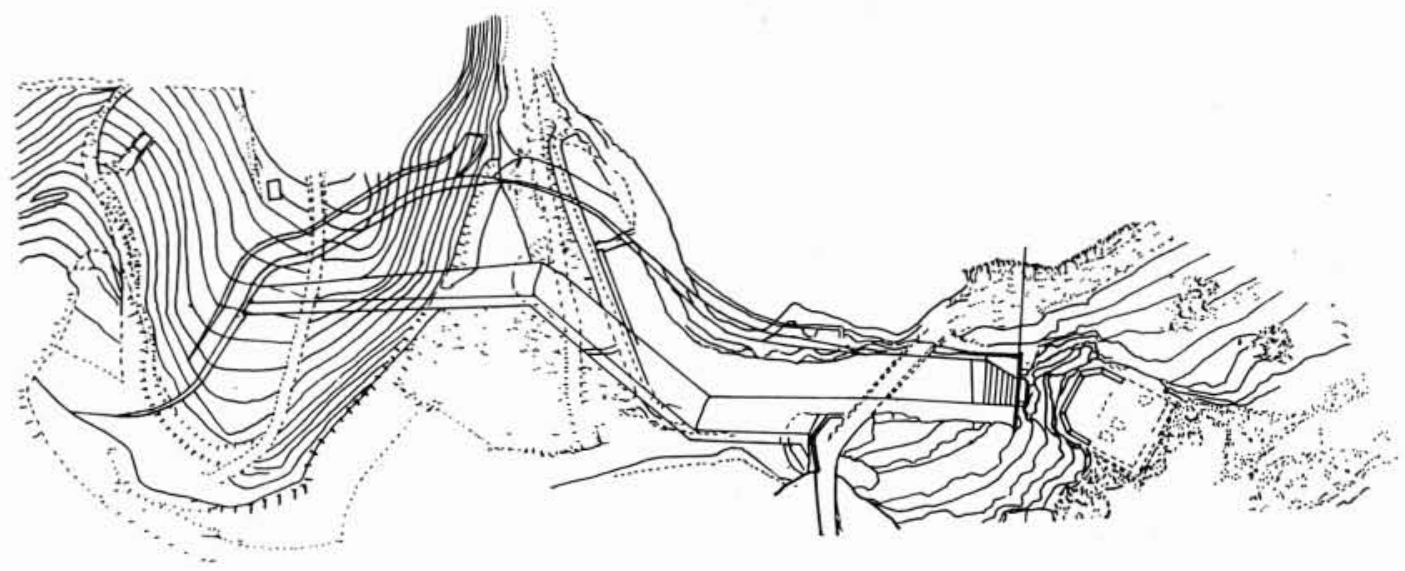

4. Evacuateur du barrage de Rochebut.

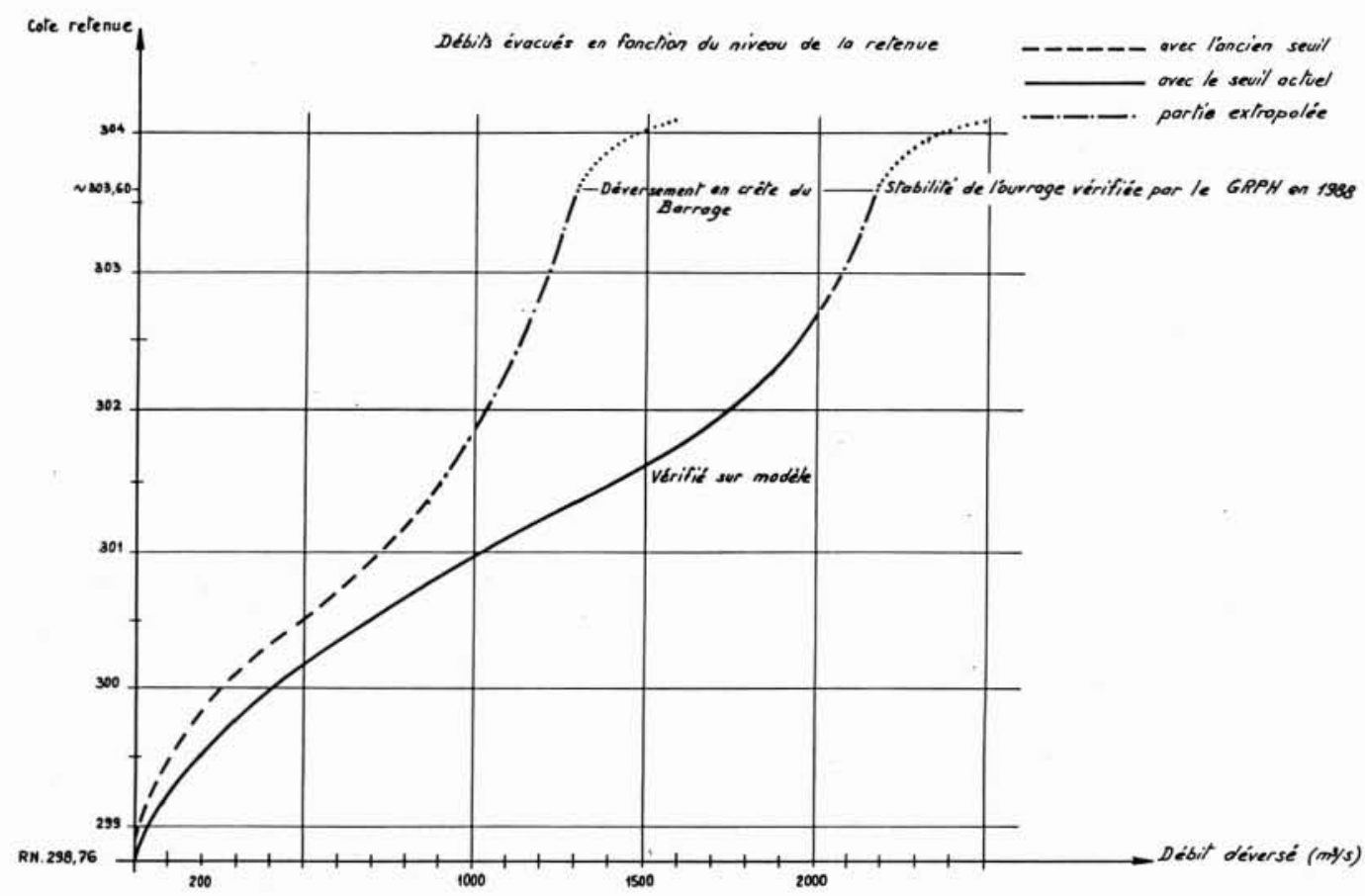

5. Seuil de Rochebut. Débits évacués en fonction du niveau de la retenue. 


\section{C - ÉVACUATEURS VANNÉS}

\section{Généralités}

L'intervention sur ces ouvrages est facilitée pour les raisons suivantes :

- les caractéristiques dimensionnelles des vannes modernes permettent le remplacement de nombreuses vannes par des matériels moins nombreux, plus grands, plus performants. Il est donc possible, dans un même environnement, d'atteindre l'accroissement de débit désiré :

- la disposition de ces matériels dans le sommet de la tranche permet de minimiser les incidences sur l'exploitation de la chute en choisissant ses périodes d'intervention, le problème restant de ne pas enfreindre les conditions de sécurité de l'ouvrage pour le passage des crues, par exemple.

Dans ce type d'ouvrage, nous citerons les exemples de Guerlédan, de Pinet, des Saints Peyres et de Castelnau comme illustration des propos précédents.

\section{Barrage de Guerlédan}

\subsection{Caractéristiques}

Situé sur le Blavet, dans le département des Côtes-duNord, le barrage de Guerlédan a été mis en service en 1931.

Il crée sur le Blavet une retenue de $49,84 \mathrm{hm}^{3}$ de capacité totale, dont $20,84 \mathrm{hm}^{3}$ utiles.

C'est un barrage poids en béton, de $206 \mathrm{~m}$ de longueur en crête. Sa hauteur maximale au-dessus de la fondation est de $54,60 \mathrm{~m}$.

Hydrologie :

- Bassin versant : $1050 \mathrm{~km}^{2}$.

- DMI centenal : $300 \mathrm{~m}^{3} / \mathrm{s}$.

— DMI millénal : $500 \mathrm{~m}^{3} / \mathrm{s}$.

\subsection{Evacuateur de crues initial}

Situé en rive droite, il est composé de :

- deux pertuis de $6 \mathrm{~m}$ de largeur, équipés de vannes STONEY de $6 \mathrm{~m} \times 3,85 \mathrm{~m}$;

- un pertuis de $6 \mathrm{~m}$ de largeur, équipé d'une vanne segment automatique de $6 \mathrm{~m} \times 3 \mathrm{~m}$, à commande par flotteur.

Les eaux évacuées par les vannes sont ramenées à la rivière par un chenal creusé dans le rocher de la rive droite et partiellement maçonné.

\section{Capacité initiale de l'évacuateur de crues}

Le débit théorique des deux vannes STONEY était de $264 \mathrm{~m}^{3} / \mathrm{s}$, celui de la vanne segment de $61 \mathrm{~m}^{3} / \mathrm{s}$, ce qui
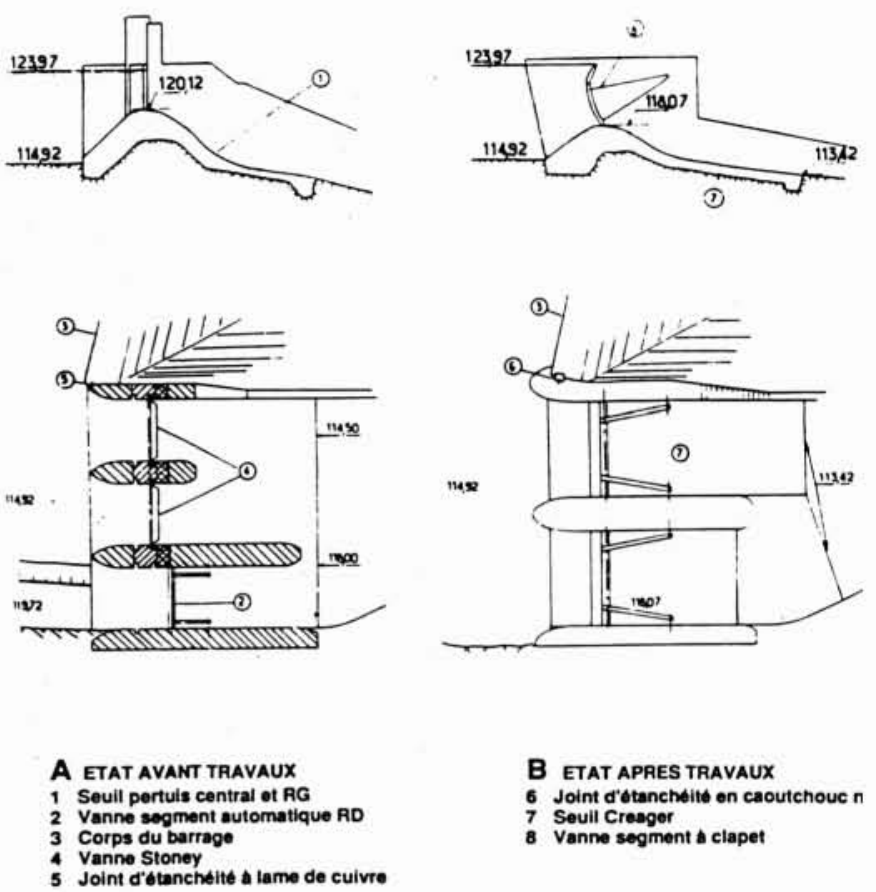

B etat apres travaux

5 Joint d'otancheite en caoutchouc Joint d'otanch Vanne segment to clapet

6. Barrage de Guerlédan. Evacuateur de crues.

conduisait à une capacité totale d'évacuation des crues de $325 \mathrm{~m}^{3} / \mathrm{s}$.

Une estimation plus fine des coefficients de débit des seuils confirmée par essais sur modèle réduit, conduisait à une capacité totale de l'ordre de $220 \mathrm{~m}^{3} / \mathrm{s}$, le lac étant à la cote de retenue normale.

\subsection{Modifications envisagées (fig. 6)}

Compte tenu des éléments exposés plus haut, et de la très faible capacité de laminage des crues offerte par la retenue, il importait d'augmenter la capacité d'évacuation de $280 \mathrm{~m}^{3} / \mathrm{s}$ afin d'assurer le passage du débit de pointe de la crue millénale sans surélévation du plan d'eau.

L'abaissement des seuils des pertuis existants en conservant les piles a été étudié sur modèle. Cette solution présentait l'avantage d'atteindre l'objectif en limitant les terrassements à réaliser.

Mais cette solution nécessitant la reprise en sous-œuvre des piles existantes afin d'assurer leur stabilité avec une charge augmentée de $2,50 \mathrm{~m}$, était relativement coûteuse et ne modernisait pas de façon radicale l'ouvrage d'évacuation. 


\subsection{Solution retenue}

Cette solution consistait à remplacer les 3 pertuis existants par deux pertuis de $9,50 \mathrm{~m}$ de largeur dont le seuil présentait un abaissement de $2,90 \mathrm{~m}$ en rive droite et de $2,05 \mathrm{~m}$ en rive gauche, équipés de vannes segments surmontés de clapets identiques. La passe rive droite fut équipée d'un seuil plat (afin de réduire les déroctages), la passe rive gauche d'un seuil en profil Creager.

Les essais sur modèle, après adaptation des nez de piles, permirent de déterminer le débit possible à la R.N.: $520 \mathrm{~m}^{3} / \mathrm{s}$ avec un écoulement correct dans le chenal aval.

Le modèle a également mis en évidence l'inégalité des débits évacués par les 2 vannes pourtant identiques. Ce fait souvent rencontré est essentiellement dû aux distorsions des champs de vitesse à l'amont des vannes dues à la topographie de la retenue.

\section{Barrage de Pinet}

Les modifications réalisées sur cet ouvrage seront largement développées dans l'exposé de M. SOYER.

\subsection{Caractéristiques}

Le barrage de Pinet est situé sur le Tarn, une trentaine de $\mathrm{km}$ à l'aval de Millau.

Barrage-poids en béton surmonté d'une route départementale.

Construction 1929.

Hauteur $40 \mathrm{~m}$ environ.

Longueur $177 \mathrm{~m}$.

Retenue de $13,2 \mathrm{hm}^{3}$.

Hydrologie :

- Bassin versant : $2677 \mathrm{~km}^{2}$.

- DMI millénal : $4400 \mathrm{~m}^{3} / \mathrm{s}$.

\subsection{Evacuateur de crues initial}

L'évacuateur de crues occupe toute la partie supérieure du barrage.

Le déversoir présente, dans sa partie haute, la forme d'un profil "Craeger ». Arasé à la cote $315,10 \mathrm{NGF}$ il est surmonté de 18 vannes-segments de $6,50 \mathrm{~m}$ de large et de $5,15 \mathrm{~m}$ de hauteur permettant d'exploiter le barrage à la cote $320 \mathrm{NGF}$.

Ces vannes sont regroupées en trois groupes de six, installés entre des piles en béton de 3,24 $\mathrm{m}$ de large. Au sein de chaque groupe, les vannes sont séparées par des fermettes à ossature métallique.

Le débit théorique total d'évacuation est de $3500 \mathrm{~m}^{3} / \mathrm{s}$.

Le projet de réfection complète de l'évacuateur de crues répond à un double objectif :

- augmentation du débit nominal,

- amélioration du passage des corps flottants.

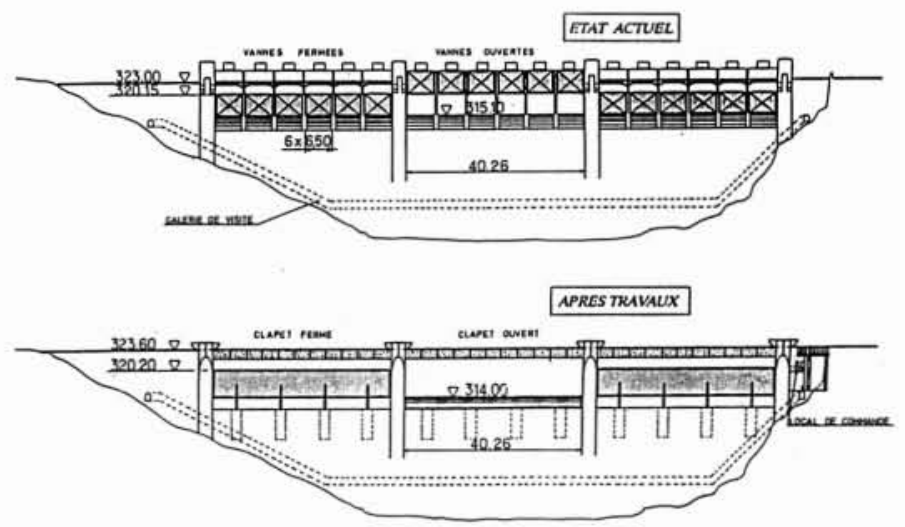

7. Elévation aval générale.

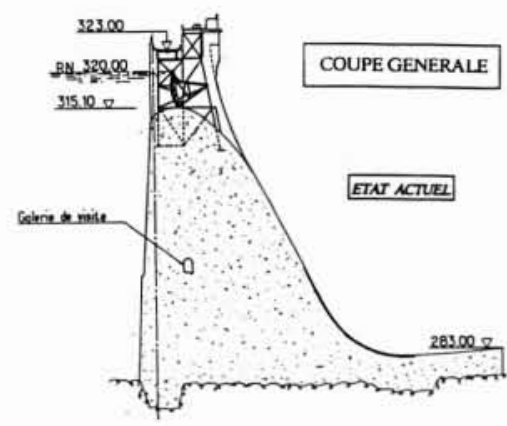

Barrage de Pinet

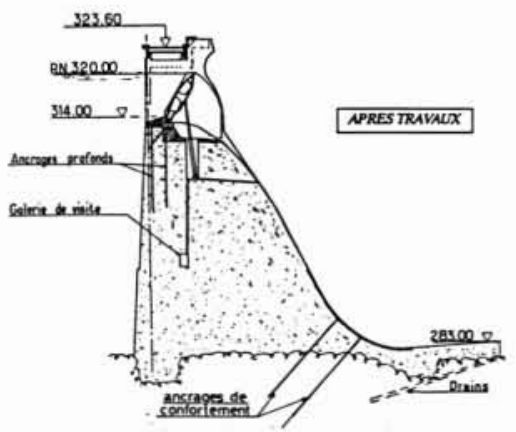

8. Coupe générale.

Remarque : actuellement, la capacité d'évacuation est en réalité bien inférieure au débit théorique de $3500 \mathrm{~m}^{3} / \mathrm{s}$ car les pertuis des vannes subissent, lors des crues, une obstruction importante par les corps flottants.

\subsection{Les modifications (fig. 7 et 8 )}

Le passage du débit théorique de $3500 \mathrm{~m}^{3} / \mathrm{s}$ à $4400 \mathrm{~m}^{3} / \mathrm{s}$, sans surélévation de la cote actuelle des plus hautes eaux (321 NGF) est réalisé par abaissement du seuil déversant.

Afin de résoudre le problème des corps flottants, les 18 vannes segments actuelles sont remplacées par 3 clapets abaissants de 40,26 $\mathrm{m}$ de largeur.

A noter que la difficulté des travaux fut notablement augmentée par les contraintes suivantes :

- conserver la libre circulation sur l'ouvrage pendant toute leur durée ;

- assurer le niveau de retenue normale pendant les périodes estivales pour des raisons touristiques. 


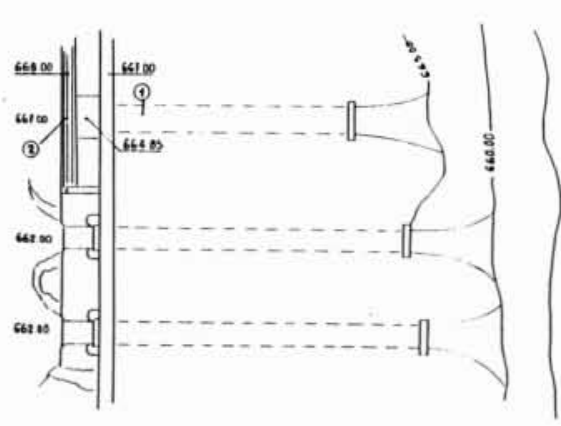

A

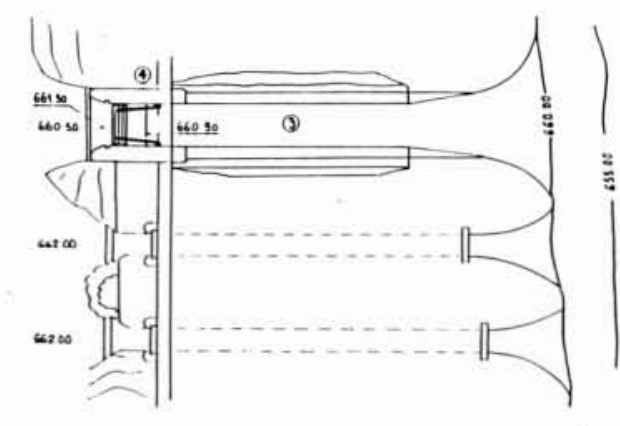

B
ETAT AVANT TRAVAUX

1 Evacuateur en Galerie

2 DEVERSOIR
ETAT APRES TRAVAUX

3 NOUVEAU CHENAL DEVACUATION DES Chues

4 VANNE SEGMENI

9. Barrage des Saints Peyres. Evacuateur de crues.

\section{Barrage des Saints Peyres}

\subsection{Caractéristiques}

Le barrage des Saints Peyres, sur l'Arn, contrôle 129 km² du bassin versant de L'Agout (Tarn).

Barrage-poids en béton.

Construction 1935.

Hauteur $60 \mathrm{~m}$ environ.

Longueur $180 \mathrm{~m}$.

Retenue de $34 \mathrm{hm}^{3}$.

Hydrologie :

- Bassin versant : $122 \mathrm{~km}^{2}$.

— DMI millénal : $450 \mathrm{~m}^{3} / \mathrm{s}$.

\subsection{Evacuateur de crues initial}

Le barrage possède actuellement les ouvrages d'évacuation suivants :

- Seuil déversant d'une longueur de $20 \mathrm{~m}$ calé à la cote 669 NGF est fermé par un clapet à flotteur pour obtenir une cote de retenue normale à 670 NGF.

- Galeries de décharge de section en fer à cheval de diamètre $3,25 \mathrm{~m}$ permettent d'évacuer un débit voisin de $220 \mathrm{~m}^{3} / \mathrm{s}$ pour une cote de retenue à $670 \mathrm{NGF}$. Chacune est fermée par une vanne-wagon, dont le seuil est calé à 660,05 NGF.

- Vidanges de fond :

- Conduite à travers le barrage

- conduite métallique de diamètre $1,30 \mathrm{~m}$,
- longueur : $49,70 \mathrm{~m}$

- Conduite de dérivation provisoire

- longueur de galerie : $46,65 \mathrm{~m}$,

— diamètre de galerie : $2,80 \mathrm{~m}$ (non revêtue),

- longueur de conduite : $96,10 \mathrm{~m}$,

— diamètre de conduite : $1,30 \mathrm{~m}$.

Débit unitaire estimé sous RN 670 NGF
- 1 vidange de fond
-1 vidange de dérivation provisoire
-1 seuil déversant
-2 évacuateurs de demi-fond

\section{TOTAL}

Sans utilisation des vidanges de fond, le débit maximal évacuable n'excède pas $251 \mathrm{~m}^{3} / \mathrm{s}$, montrant ainsi l'insuffisance des ouvrages actuels, face à une crue de projet de $450 \mathrm{~m}^{3} / \mathrm{s}$.

\subsection{Les solutions envisagées (fig. 9)}

Plusieurs solutions ont été envisagées seules ou en combinaison :

- utilisation du laminage en admettant une légère surélévation des PHE,

- installation d'une vanne dans le seuil sous le clapet,

- abaissement du seuil,

- redimensionnement des vidanges de fond,

- surcreusement de la galerie de seuil avec mise en charge,

- évacuateur en tranchée avec vanne de surface. 


\subsection{Le projet retenu}

Après examen, les solutions faisant intervenir les vidanges de fond ou une surélévation de la cote des PHE ont été éliminées.

Le projet retenu est l'évacuateur complémentaire en tranchée avec vanne de surface.

Les arguments favorisant ce choix sont les suivants :

- sécurité de la consigne de crue,

— vétusté et faible intérêt du seuil à clapet,

- faible indisponibilité pendant les travaux,

- sécurité de fonctionnement.

Deux types de vannes de surface peuvent être envisagés : vanne-wagon ou vanne-segment.

La solution à vanne-segment est la plus économique et a été adoptée.

\section{Le barrage de Castelnau}

\subsection{Caractéristiques}

Le barrage de Castelnau est situé sur le Lot sur un col séparant la vallée du Lot et du Mossau.

Barrage-poids en béton.

Construction 1951.

Hauteur $60 \mathrm{~m}$ environ.

Longueur $180 \mathrm{~m}$.

Retenue de $40 \mathrm{hm}^{3}$.

Hydrologie :

- Bassin versant : $1050 \mathrm{~km}^{2}$.

- DMI millénal : $2080 \mathrm{~m}^{3} / \mathrm{s}$.

\subsection{Evacuateur de crues initial}

Deux seuils déversants de $15 \mathrm{~m}$ de largeur, fermés par 2 vannes secteur de $9,10 \mathrm{~m}$ de hauteur, ferme de col entre la vallée du Lot et du Mossau à l'opposé du barrage.

La capacité d'évacuation totale est de $1480 \mathrm{~m}^{3} / \mathrm{s}$ sous $\mathrm{RN}$, soit un déficit de $600 \mathrm{~m}^{3} / \mathrm{s}$ par rapport à la crue de projet.

\subsection{Les solutions envisagées (fig. 10)}

Deux solutions ont été envisagées :

- rétablir l'ancienne dérivation provisoire,

- réaliser un ouvrage neuf déversant directement dans la vallée du Mossau.

La première solution conduisait à un réalésage délicat de l'ancienne galerie, et le passage des crues dans une gorge très resserrée, aurait conduit à une surélévation du niveau de l'eau excessive au droit de l'usine et à un risque d'érosion de sa route d'accès.

Il a donc été choisi de réaliser un ouvrage neuf, indépendant des ouvrages existants comportant :

- une prise d'eau en surface, équipée d'une vanne secteur de $11,80 \mathrm{~m}$ de large et $8,75 \mathrm{~m}$ de hauteur ;

- une galerie de $7 \mathrm{~m}$ de diamètre et $330 \mathrm{~m}$ de longueur blindée sur ses 30 premiers mètres, revêtue ailleurs ;

- un ouvrage aval destiné à dissiper l'énergie en sortie de galerie en étalant le jet et en le projetant à une distance suffisante pour éviter l'érosion de l'ouvrage.

L'ensemble de ces ouvrages ont été vérifiés sur modèle réduit, tant vis-à-vis de leur débitance que sur l'aspect qualitatif de leur fonctionnement, en particulier la cuillère aval.

Les travaux actuellement en cours permettront une mise en service du nouvel ouvrage en 1992.

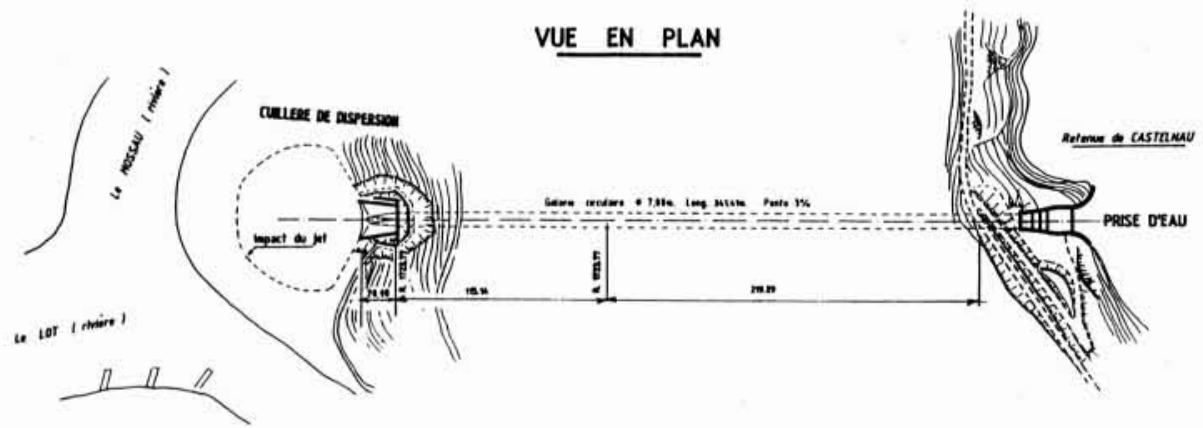

PROFIL EN LONG

10. Castelnau.

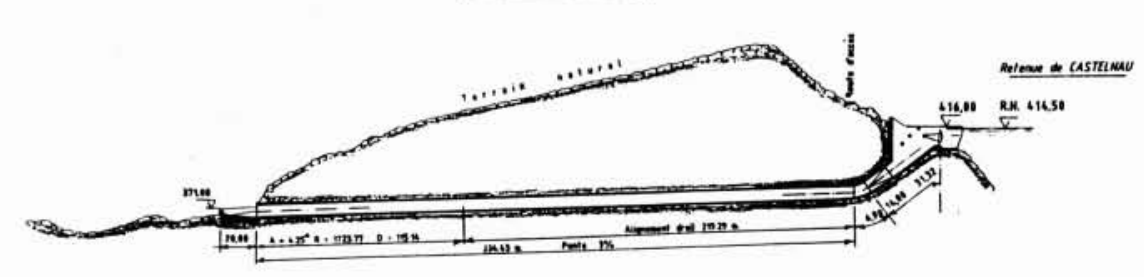




\section{D - CONCLUSIONS}

Ces quelques exemples montrent bien la spécification de chaque cas, avec ses contraintes propres :

- l'interruption de la circulation impossible à Pinet et Rochebut est acceptable sur les autres ouvrages;

- l'évacuation des corps flottants, problème majeur à Pinet dans le choix du nouveau matériel n'est pas déterminant au St-Peyres ou Castelnau ;

- réfection de la totalité des matériels d'évacuation de crue, ou ajout d'un organe complémentaire ;

- aménagement des ouvrages existants comme à Thuries ou reconstruction totale comme à Queuilles ou Rochebut ;

- etc...,

qui conduisent à des solutions bien différentes tant dans la conception et dans le mode de réalisation des travaux que dans leur bilan économique.
Il faut néanmoins noter les points communs à ces différents exemples et caractéristiques de l'approche d'EDF :

- l'appel au modèle réduit, au stade de l'étude. Elément majeur dans la définition des formes, le dimensionnement, l'étude de l'évacuation des corps flottants, impact des jets, etc..., permet au projeteur d'appréhender physiquement le problème dans son ensemble ;

- le maintien des performances en capacité de stockage des barrages concernés (un abaissement des seuils existants et donc de la retenue normal aurait pu se concevoir dans certains cas mais au détriment de la capacité en exploitation courante).

Ces quelques cas montrent aussi le prix de la sécurité pour reculer le danger face à un risque aléatoire.

\section{Bibliographie}

Certains éléments du document ci-dessus sont extraits des documents suivants :

Millet J.C., Chambon J., Soyer G., Lefèvre C. $-16^{\mathrm{e}}$ CIGB à San Francisco 1988. Augmentation de la capacité des ouvrages d'évacuation de divers barrages.

Réaménagement de l'évacuateur de crues du barrage de Guerlédan. Texte de l'exposé présenté au stage BETGGB du 23/10/87.

Devernay J.M. - Rénovation de l'évacuateur de crues du barrage de Pinet. Revue de l'énergie $n^{\circ} 410$, mars-avril 1989.

Devernay J.M. - Barrage de Pinet. Réfection de l'évacuateur de crue. Exposé à la conférence Water Power du 19 au 21/10/87 Strasbourg. 